\title{
Dexamethasone implant (Ozurdex®) migration to anterior chamber through a congenital iris coloboma: A case report
}

Yoav Glidai ( $\square$ yoavglidai@gmail.com )

Tel Aviv University Sackler Faculty of Medicine https://orcid.org/0000-0002-3269-994X

Shulamit Schwartz

Tel Aviv Sourasky Medical Center

Eyal Cohen

Tel Aviv Sourasky Medical Center

\section{Case report}

Keywords: Ozurdex®, implant, migration, iris coloboma, surgical technique

Posted Date: April 17th, 2019

DOI: https://doi.org/10.21203/rs.2.9226/v1

License: (c) (i) This work is licensed under a Creative Commons Attribution 4.0 International License.

Read Full License 


\section{Abstract}

Background: Ozurdex ${ }^{\circledR} 0.7 \mathrm{mg}$ (dexamethasone $0.7 \mathrm{mg}$ implant, Allergan, Dublin, Ireland), an intra-vitreal biodegradable implant indicated for cystoid macular edema due to variable causes. One of its known complications is implant migration to the anterior chamber, causing corneal edema that in some cases might be irreversible. Reported risk factors for device migration are open or defective lens capsule and prior history of vitrectomy. We present a case of dexamethasone implant migration through a congenital iris coloboma in a pseudophakic patient with intact lens capsule. Case presentation: 56-year-old pseudophakic man with a history of congenital iris coloboma, myopia, retinal tears and a Branch Retinal Vein Occlusion (BRVO) with subsequent Cystoid Macular Edema (CME) which was resistant to anti-VEGF medications but responsive to corticosteroids. He presented with sudden painless decreased vision in his left eye, eight weeks after dexamethasone implant (Ozurdex $\left.{ }^{\circledR}\right)$ injection to the same eye. Upon presentation he was diagnosed with corneal edema caused by anterior chamber migration of the implant. He was referred for immediate surgical intervention and the implant was extracted, with the edema completely resolved within two weeks post-op. Conclusions: This is the first case reported of implant migration through an iris coloboma in the setting of intact posterior capsule. In addition, we describe a novel approach for implant removal surgery that is simple and efficient. This case sheds a light on another risk factor that should be taken into consideration in the management of this patient population.

\section{Background}

Ozurdex® $0.7 \mathrm{mg}$ (dexamethasone $0.7 \mathrm{mg}$ implant, Allergan, Dublin, Ireland) is a $0.46 \mathrm{~mm}$ diameter and 6 $\mathrm{mm}$ length biodegradable implant, injected into the vitreous cavity for the treatment of macular edema due to several conditions, among them cystoid macular edema (CME) due to branch or central vein occlusion (1). In the recent years, as the clinical indications for this treatment has expanded, episodes of implant migration to the anterior chamber have been reported. The main risk factors for device migration are open or defective lens capsule and prior history of vitrectomy $(2,3)$. Recently, Stepanov et al. and Stelton et al. have reported of implant migration in patients with an Iris defect $(4,5)$, suggesting it could be the migration route. It is of note, that all the reported cases had, in addition to the Iris defect, a defective lens capsule. Migration in the setting of intact posterior capsule have also been reported, in which migration was speculated to occur through weak or ruptured zonules (6).

When anterior chamber migration occurs, and corneal edema begins to develop, it is crucial to remove the implant promptly. If not taken out expeditiously, implant migration can lead to permanent corneal compromise $(2,3)$. We present a case of dexamethasone implant migration through a congenital iris coloboma in a pseudophakic patient with intact lens capsule.

\section{Case Presentation}

A 56-year-old pseudophakic man presented with sudden painless decreased vision in his left eye (LE), eight weeks after dexamethasone implant (Ozurdex $\left.{ }^{\circledR}\right)$ injection to the aforesaid eye. He was treated with 
Ozurdex® implant for macular edema following branch retinal vein occlusion (BRVO) that occurred four years prior his presentation; this was his second Ozurdex ${ }^{\circledR}$ implantation. His additional past ocular history included myopia in both eyes; right 11D and left 2D, with laser refractive correction surgery done in his right eye (RE), congenital inferior iris coloboma in his LE and history of several retinal tears in both eyes treated with laser retinopexy and cryotherapy. In addition, a year after the BRVO occurred he underwent uneventful cataract surgery to his LE with posterior chamber intra ocular lens (PCIOL) implantation.

The patient had experienced BRVO in his LE with subsequent macular edema four years prior his presentation. When he began retinal clinic follow-up and treatment at our center his best corrected visual acuity (BCVA) was 20/20 and 20/200 at his right and left eye, respectively. A macular optical coherence tomography (OCT) of his LE demonstrated CME with elevated central macular thickness (CMT) of 465 $\mu \mathrm{m}$. During his follow-up he was treated with intravitreal anti VEGF agents which included: Bevacizumab, Ranibizumab and Aflibercept injections, all of which had no significant effect on the macular edema. As a second line therapy Triamcinolone was injected intravitreally, resulting in partial improvement of the macular edema with a fair amount of fluid absorbed. Four weeks post injection, macular OCT showed a decrease in CMT from $507 \mu \mathrm{m}$ to $440 \mu \mathrm{m}$. As this improvement suggested good response to corticosteroid treatment, he was qualified for Ozurdex ${ }^{\circledR}$ implant injection. One month following the first implant insertion we witnessed a significant improvement in his LE macular edema, with an OCT scan showing a decrease in CMT from 405 to $311 \mu \mathrm{m}$. Eight weeks before his presentation he underwent a second Ozurdex ${ }^{\circledR}$ injection with similar response. In his last retina clinic visit, one week before presentation, a BCVA of 20/200 was recorded in his LE.

Our patient presented with sudden decreased "hazy" vision in his LE; visual acuity was 1/36 and 20/20 in his left and right eye, respectively. Slit-lamp examination revealed central and inferior stromal corneal edema in his LE. The anterior chamber (AC) was deep, with the dexamethasone implant lying in the inferior angle touching the inferior cornea (Figure 1). Iris coloboma was open and PCIOL was correctly situated in the BAG with an intact posterior capsule, LE intraocular pressure measured $18 \mathrm{mmHg}$.

After ruling out other causes for corneal edema, it was determined that that the cause was implant migration. A literature review on such cases was preformed, concluding the prompt extraction of the implant is needed to avoid persistent corneal decompensation. The patient was than referred for surgical intervention within 24 hours from presentation.

The operation comprised of making two paracentesis 180 degree apart; introducing an AC maintainer of basic salt solution through one and a spatula gently opening the second paracentesis directly across. Due to pressure gradient and stream created by the maintainer the dexamethasone implant was navigated toward the second paracentesis and easily ejected from the AC. following the extraction of the implant an intracameral Cefuroxime was injected and incision hydration was made.

One day post-op on eye examination the corneal edema was still evident, anterior chamber was deep and quiet, intra ocular pressure was $15 \mathrm{mmHg}$. On examination two weeks post-op, the patients' LE BCVA was 
20/400 and corneal edema had resolved completely. As the steroidal treatment was interrupted by implant removal, on macular OCT we witnessed a deterioration in the macular edema with CMT increasing from $342 \mu \mathrm{m}$ to $477 \mu \mathrm{m}$. After careful deliberation (elaborated in "Discussion") we found that the best course of action would be renewal of the previous treatment. The patient underwent a third Ozurdex ${ }^{\circledR}$ injection to his LE. In a follow-up up to this day, 4 months post injection, there hasn't been recurrence of implant migration.

\section{Discussion}

Ozurdex ${ }^{\circledR}$ implant anterior chamber migration is a rare complication that can lead to permanent corneal decompensation. In a retrospective study, Khurana and colleagues showed that early removal of the implant is essential for corneal recovery (3). The "classical" risk factors for implant migration identified in that pivotal study were (1) History of pars plana vitrectomy, and (2) open or defective lens capsule. In a recent review article by Rahimy et al. two additional risk factors was recognized: (3) Iris defect (patent laser iridotomy or surgical iridectomy) and (4) zonular dehiscence (from previous trauma or cataract surgery) (2). Kocak et al. was the first to describe implant migration in the setting of intact posterior capsule, assuming the culprit were weak zonules (6). Two cases have been reported about iris defects being the route of passage. First was Stelton et al., who described a patient with PCIOL and a disrupted posterior capsule that in addition had a large peripheral inferior iridectomy (5). Later, Stepanov et al. described a patient that underwent inferior iridectomy due to silicone oil insertion during pars plana vitrectomy for treating retinal detachment, that was later on implanted with iris claw anterior chamber IOL (Artisan)(4). Although it is fairly likely that in both cases the implant migrated through the lris defects, both patients also had the "classical" risk factors (prior PPV and disrupted lens capsule). In the presented case, the patient had intact posterior capsule. As discussed by Stepanov et al., an iris defect that is situated in the inferior iris (as in our case) is more susceptible be a route of passage for an implant, as it usually sinks to the bottom of the Vitreous cavity (4).

Congenital iris colobomas arise from incomplete closure of the embryonic fissure during fetal development. A "typical" congenital iris coloboma is located at the inferonasal quadrant and therefore must be considered a risk factor for $\mathrm{AC}$ dexamethasone implant migration, with this being the first report of Ozurdex ${ }^{\circledR}$ implant migration through an iris coloboma. It is essential to inform these patients of having an increased risk for implant migration and the importance of seeking an immediate medical consultation with any sudden change of vision as early removal of the implant increases corneal recovery rates (3).

Following the migrating implant removal, we faced exacerbation of the CME condition. Our team of experts came together for convergent thinking process with several alternatives considered. First option considered was to reinitiate injections of anti VEGF agents. However, As mentioned earlier, our patient has already exhausted all anti-VEGF treatment options therefore repeating trial with one of these drugs would probably be infertile. Second alternative considered was implanting a different intraocular steroidal implant. Fluocinolone acetonide 0.59mg (Retisert, Bausch \& Lomb, Rochester, New York, USA) and 
fluocinolone acetonide $0.19 \mathrm{mg}$ (Iluvien, Alimera Sciences, Alpharetta, Georgia, USA) are two steroidal implants available for intraocular use. Although lluvien is designed to provide sustained therapy for a longer period of time, it is also free-floating, but smaller in size than Ozerdex, making it even more susceptible for implant migration. Only two reports of lluvien migration have been published, with two patients suffering from consequent corneal edema $(7,8)$. Retisert, despite being anchored to the sclera, has also been reported to dislocate to the $\mathrm{AC}$, inducing corneal edema, possibly resulting from medication reservoir dissociation from its sutured strut (9-11). Furthermore, we considered iris coloboma repair (Pupilloplasty). Despite being a tempting alternative, pupilloplasty is an additional AC surgery, which involves a considerable risk to a cornea that has already gone through a considerable amount of procedures thus jeopardizing it again. At that point, our decision was to recommend repeating Ozurdex ${ }^{\circledR}$ injection since this treatment had a substantial effect on the patient's CME and improved his VA. We concluded that any other alternative would carry either the same or a greater risk to the patient's eye. The patient accepted our recommendation and underwent a third Ozurdex ${ }^{\circledR}$ implantation. The patient was instructed to return at any sudden change of vision. As mentioned, in a follow-up of 4 months post injection, implant migration had not recurred.

\section{Conclusion}

We have presented a case of dexamethasone implant migration to the AC through a congenital coloboma, resulting in corneal edema. This is the first case reported of implant migration in the setting of intact posterior capsule. We described a novel approach for implant removal surgery that is simple, efficient and cost-effective. Physicians should inform patients with iris defects of the risk of implant migration. It is eminent to emphasize that at any decrease or change in vision, patient should seek urgent medical attention.

\section{List Of Abbreviations}

CME - Cystoid macular edema

BRVO - branch retinal vein occlusion

PCIOL - Posterior chamber intra-ocular lens

CMT - Central macular thickness

OCT - optical coherence tomography

VEGF - Vascular endothelial growth factor

$\mathrm{AC}$ - anterior chamber

\section{Declarations}


Ethics approval and consent to participate: the patient gave his informed consent to this publication.

Consent for publication: Written informed consent was obtained from the patient for publication of this article and any accompanying images. A copy of the written consent is available for review by the Editor of this journal.

Availability of data and material: not applicable.

Competing interests: The authors declare that they have no competing interests.

Funding: no financial support was received for the submission.

Authors contribution: YG contributed to manuscript conception, design and acquisition. EC and SS contributed to acquisition, analysis and interpretation of data. All authors have been involved in drafting the manuscript or revising it critically and have given final approval of this version to be published and agreed to be accountable for all aspects of the work.

Acknowledgments: not applicable.

\section{References}

1. Haller JA, Bandello F, Belfort R, Jr., Blumenkranz MS, Gillies M, Heier J, et al. Randomized, shamcontrolled trial of dexamethasone intravitreal implant in patients with macular edema due to retinal vein occlusion. Ophthalmology. 2010;117(6):1134-46.e3.

2. Rahimy E, Khurana RN. Anterior segment migration of dexamethasone implant: risk factors, complications, and management. Current opinion in ophthalmology. 2017;28(3):246-51.

3. Khurana RN, Appa SN, McCannel CA, Elman MJ, Wittenberg SE, Parks DJ, et al. Dexamethasone implant anterior chamber migration: risk factors, complications, and management strategies. Ophthalmology. 2014;121(1):67-71.

4. Stepanov A, Codenotti M, Ramoni A, Prati M, Jiraskova N, Rozsival P, et al. Anterior chamber migration of dexamethasone intravitreal implant (Ozurdex(R)) through basal iridectomy (Ando) in a pseudophakic patient. European journal of ophthalmology. 2016;26(3):e52-4.

5. Stelton CR, Townsend J, Peterson LT, Khurana RN, Yeh S. Surgical Management of Anterior Chamber Migration of a Dexamethasone Intravitreal Implant. Ophthalmic surgery, lasers \& imaging retina. 2015;46(7):756-9.

6. Kocak N, Ozturk T, Karahan E, Kaynak S. Anterior migration of dexamethasone implant in a pseudophakic patient with intact posterior capsule. Indian journal of ophthalmology. 2014;62(11):1086-8. 
7. Papastavrou VT, Zambarakji H, Dooley I, Eleftheriadis H, Jackson TL. OBSERVATION: FLUOCINOLONE ACETONIDE (ILUVIEN) IMPLANT MIGRATION INTO THE ANTERIOR CHAMBER. Retinal cases \& brief reports. 2017;11(1):44-6.

8. El-Ghrably IA, Saad A, Dinah C. A Novel Technique for Repositioning of a Migrated ILUVIEN((R)) (Fluocinolone Acetonide) Implant into the Anterior Chamber. Ophthalmology and therapy. 2015;4(2):12933.

9. Almeida DR, Chin EK, Mears K, Russell SR, Mahajan VB. Spontaneous dislocation of a fluocinolone acetonide implant (Retisert) into the anterior chamber and its successful extraction in sympathetic ophthalmia. Retinal cases \& brief reports. 2015;9(2):142-4.

10. Chang PY, Kresch Z, Samson CM, Gentile RC. Spontaneous Dissociation of Fluocinolone Acetonide Sustained Release Implant (Retisert) with Dislocation into the Anterior Chamber. Ocular immunology and inflammation. 2015;23(6):454-7.

11. Akduman L, Cetin EN, Levy J, Becker MD, Mackensen F, Lim LL. Spontaneous dissociation and dislocation of Retisert pellet. Ocular immunology and inflammation. 2013;21(1):87-9.

\section{Figures}




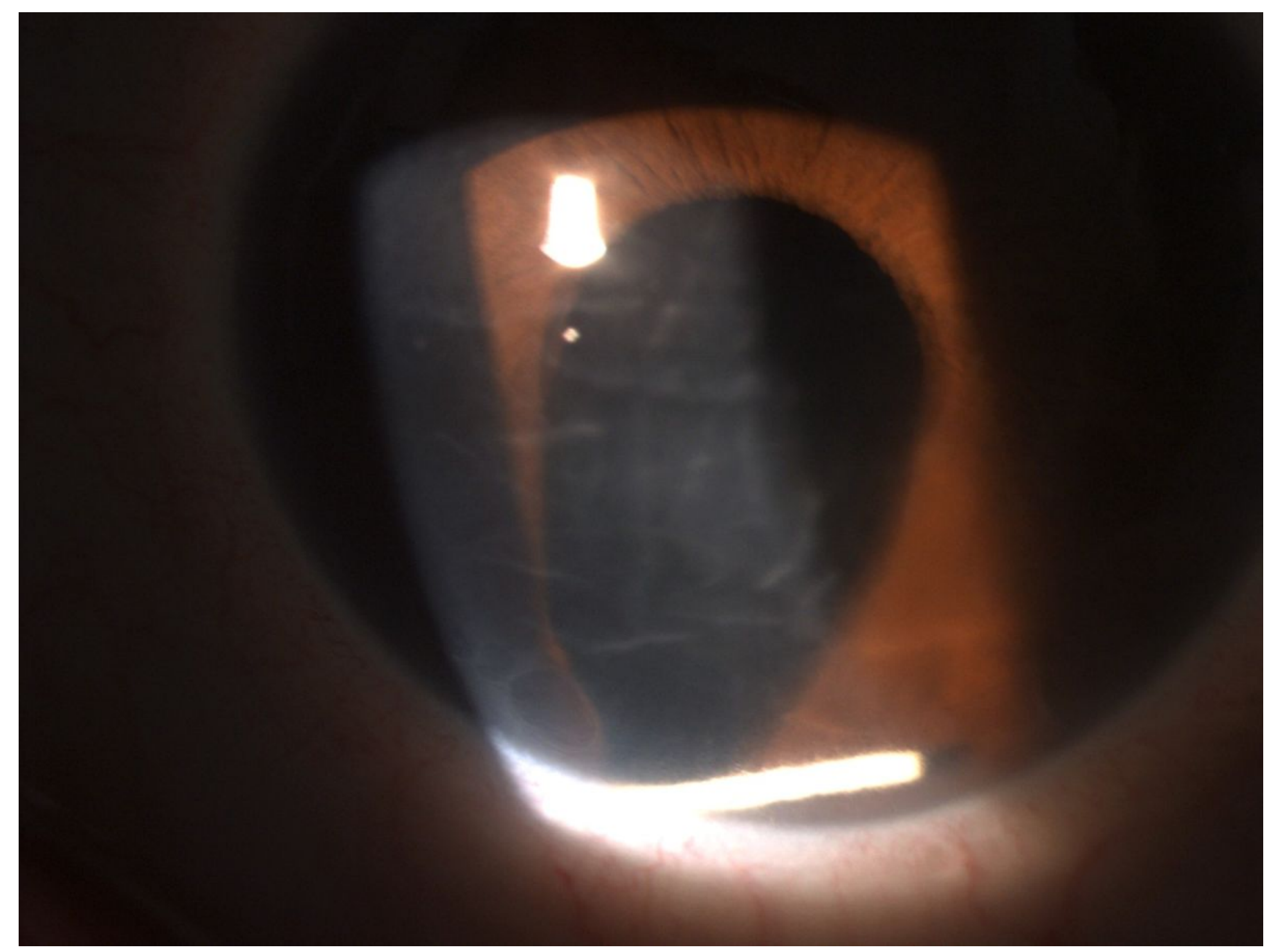

\section{Figure 1}

Slit lamp biomicroscopy capturing dexamethasone implant dislocated through inferior iris coloboma to anterior chamber with corneal edema and Descemet folds.

\section{Supplementary Files}

This is a list of supplementary files associated with this preprint. Click to download.

- supplement1.pdf 